\title{
Straight Through Hole Drilling in Machinable Ceramics
}

\author{
Susumu Nakamura $^{* 1}$, Takumi Miura ${ }^{* 2}$ and Masaya Tsuta $^{* 2}$ \\ ${ }^{* 1}$ Department of Electrical and Electronic Systems Engineering \\ Nagaoka National College of Technology, 888 Nishikatakai, Nagaoka, Niigata 940-8532, Japan \\ E-mail: snaka@nagaoka-ct.ac.jp \\ ${ }^{* 2}$ Electrical \& Mechanical Systems Engineering Advanced Course \\ Nagaoka National College of Technology, 888 Nishikatakai, Nagaoka, Niigata 940-8532, Japan
}

\begin{abstract}
This paper presents a unique through hole forming method in machinable ceramics using a flowable backing material during nanosecond pulse UV laser radiation. While processing through holes, the flowable backing material is arranged to be in contact with the back side of machinable ceramics, i.e., surface of laser beam's exit side. The laser beam which penetrated machinable ceramics strikes the flowable backing material and superheated plasma is generated by the laser beam's absorption into the flowable backing material around exit hole. The induced plasma is elevated with the flowable backing material from the back side of machinable ceramics to the front side of that by a capillary phenomenon, and the shape of through holes changed from tapered shape to straight shape by the elevation of plasma. Through this technique, we are able to make substrate for a probe card which has straight shape holes with narrow pitch.
\end{abstract}

DOI: $10.2961 / \mathrm{jlmn} .2015 .01 .0018$

Keywords: Machinable ceramics, Straight through hole, Drilling, Nanosecond UV laser, Prove card

\section{Introduction}

In a through hole drilling process using laser beam, it is generally known that the diameter of through hole on the laser beam exit side of processing target is smaller than the incidence side, i.e., the hole is formed in a so-called tapered shape due to the characteristics of the laser beam. However, a through hole having straight shape or inverse tapered shape is required depending on the application purpose of the hole. The hole which has straight shape or inverse tapered shape is formed by using a beam rotator device. However the beam rotator device is not widely used because of high cost. We proposed through hole processing with the flowable backing material to control the shape of through hole. We applied this technic to process straight through holes on resin materials [1,2].

In this study, we applied this technic on machinable ceramics. Machinable ceramics which has straight through holes is able to be used as substrate for a probe card in bath contact method. Figure 1 shows the schematic illustration of probe card. The probe card is a tool for testing semiconductors used at wafer test to check quality of IC or LSI in the first process of semiconductor manufacturing [3]. In the manufacturing process of semiconductors such as IC and LSI, many IC chips are fixed on a wafer. Wafer is diced and the diced chips are incorporated into packages. Before hundreds of IC chips fixed on a wafer are diced into chips of thickness few square millimeters, wafer test is performed to check quality of IC chips. At wafer test, the needles of probe make contact with the bonding pads of IC chips or LSI chips fixed on a wafer. After that, the electric characteristics are measured to check the quality of IC chips. The probe card is a crucial tool for testing semiconductors during wafer test. A lot of probes of several ten micrometers in diameter go through the probe card to contact between the electronic test system and the semiconductor wafer. Therefore it is necessary for the probe card to have the through holes of straight shape that the probes go through with an appropriate clearance. Among numerous existed methods of hole production, mechanical drilling [4] and laser machining [5] are most widely used. However, as the dimension of the hole decreases, choice of process becomes limited. There are problems of breaking and abrasion of a drill bit with the mechanical drilling when processing hole diameter become lesser than $100 \mu \mathrm{m}$. By selecting the right laser, fine micron-sized features can be produced very economically. Pulsed lasers allow the user to control the amount of energy delivered to the target material carefully, making it possible to control a laser drilling process precisely.

The objective of this study is to machine arrays of reproducible small straight holes having a diameter less than $100 \mu \mathrm{m}$ in machinable ceramics using nanosecond pulse UV laser. Machinable ceramics are materials that are easy to machine using normal machining tool such as lathe or machining center. Machinable ceramics are suitable for use in important applications that require materials of high thermal shock resistance, high chemical stability and high reactivity at high temperatures. Main applications of machinable ceramics are production parts for semiconductor manufacturing equipment, liquid crystal manufacturing equipment and electrical insulation.

In this paper, we present a unique through hole forming method. This unique method can easily control the shape of a through hole, e.g., formation of a through hole having a straight shape in which an exit hole diameter on the laser beam exit side of the processing target is equal to the entrance hole diameter on the incident side of that. By using this technique, we will be able to make the substrate 
for the probe card which has the holes of straight shape with narrow pitch.

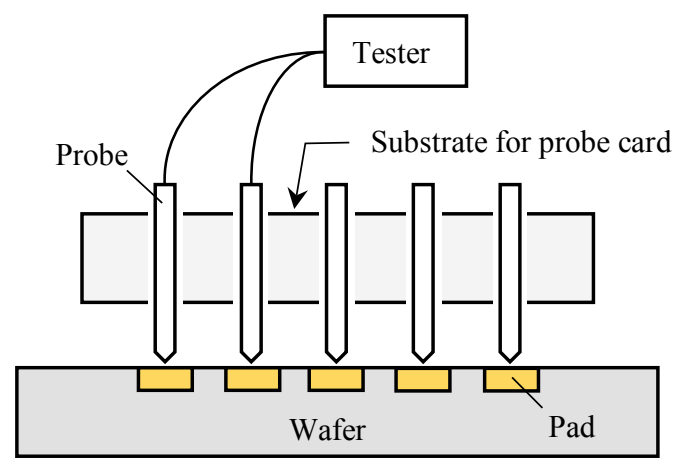

Figure 1 Schematic illustration of probe

\section{Experimental setup}

The laser used is Awave-266 (Advanced Optowave Corporation), a DPSS Q-switched $\mathrm{Nd}: \mathrm{YVO}_{4}$ laser with a forth harmonic wavelength of $266 \mathrm{~nm}$. This laser operates in TEM00 mode hence the beam has a Gaussian-distributed intensity profile. The pulse duration is less than $10 \mathrm{~ns}$ and the maximum pulse energy that can be achieved at the target surface in our setup is approximately $200 \mu \mathrm{J}$ at $5 \mathrm{kHz}$ pulse repetition rate.

To machine an array of through holes in the processing target using Awave-266, we used galvanometer scanner system with full digital control (Y-E Data, Miramotion) and a $\mathrm{f} \theta$ lens with a focal length of $53.5 \mathrm{~mm}$. In this study, trepanning drilling was adapted to make through holes of diameter less than $100 \mu \mathrm{m}$. The optical path of the laser beam from Awave-266 was changed by beam steering mirrors, then, the laser beam was expanded and linear polarization of that was formed into circular polarization with a zero-order quarter wavelength plate. The laser beam with circular polarization was controlled by $\mathrm{XY}$ axis mirrors within the galvanometer scanner system and focused onto the processing target. Here, a focal point was set to the front side of the processing target which means the laser beam incidence side. The processing target was mounted on XYZ motorized linear translation stages. Focus position of the laser beam relative to the target surface was controlled by the $\mathrm{Z}$ stage and fixed during the UV laser radiation. The diameter of focus spot was measured to be $19 \mu \mathrm{m}$ by using the burnt pattern of sample.

\section{Ultraviolet laser beam of $266 \mathrm{~nm}$}

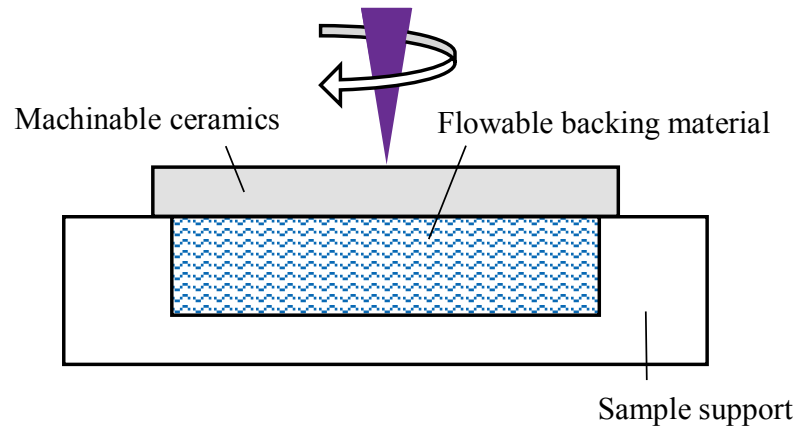

Figure 2 Schematic illustration of laser irradiation part.
Figure 2 shows the schematic illustration of laser irradiation part. Commercially available machinable ceramics, Photoveel II-s, with a thickness of $0.5 \mathrm{~mm}$ was used as the processing target. Photoveel II-s is composed by boron nitride and fine ceramics which realize the compatibility of high mechanical strength and machinability [6]. Photoveel II-s has special characteristic which is similar to thermal expansion of silicon. While processing through holes, three types of flowable backing materials, a diluted PVA glue, tap water and glycerin, each with $1 \mathrm{~mm}$ in thickness were arranged separately to be in contact with the back side of Photoveel II-s, i.e., the surface of laser beam exit side in order to obtain expanding effect of the exit hole diameter. In our previous study, the PVA glue led to the best results to make straight through holes in resin materials [2]. Therefore we used the solution obtained by diluting the PVA glue with tap water for this study. Tap water and glycerin were used for comparative experiments.

\section{Experimental results and discussion}

Figure 3 shows optical photographs of (a) cross section of through holes drilled by trepanning in Photoveel II-s having the thickness of $0.5 \mathrm{~mm}$, (b) entrance hole and (c) exit hole. In this figure, left part of the wall is the entrance, while right side is exit of the laser beam. The laser beam was scanned along a periphery of the hole but the flowable backing material was not used in this situation. Thus, we can say that this is normal trepanning drilling. The pulse

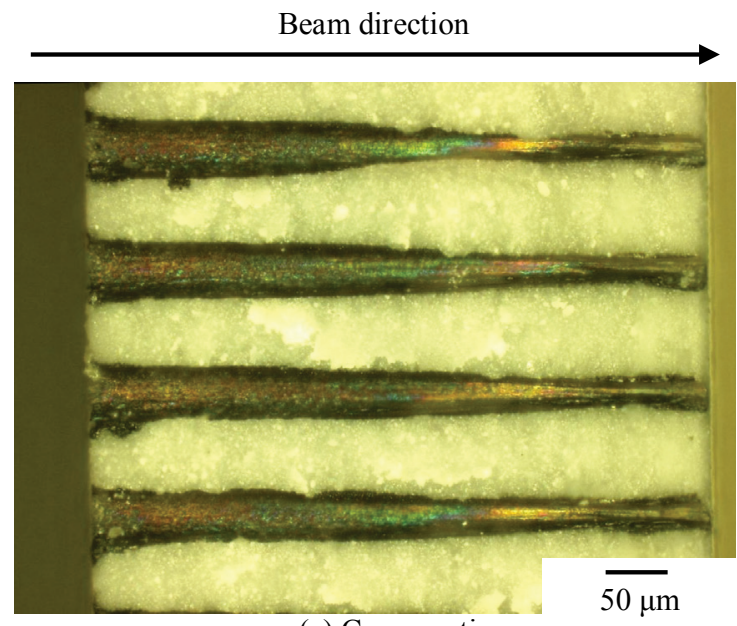

(a) Cross section

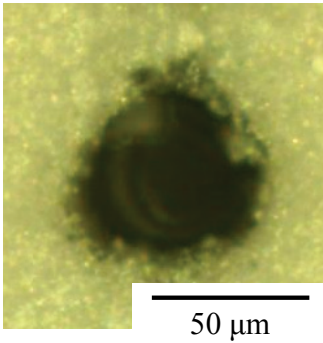

(b) Entrance hole

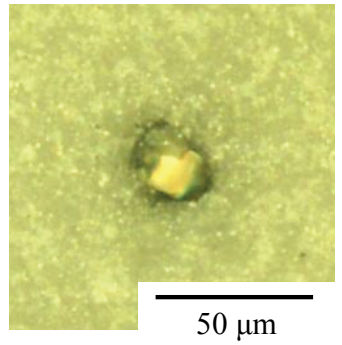

(c) Exit hole
Figure 3 Optical photographs of (a) cross section of through holes drilled in Photoveel II-s having the thickness of $0.5 \mathrm{~mm}$, (b) entrance hole and (c) exit hole. Through holes were formed without using the flowable backing material. 


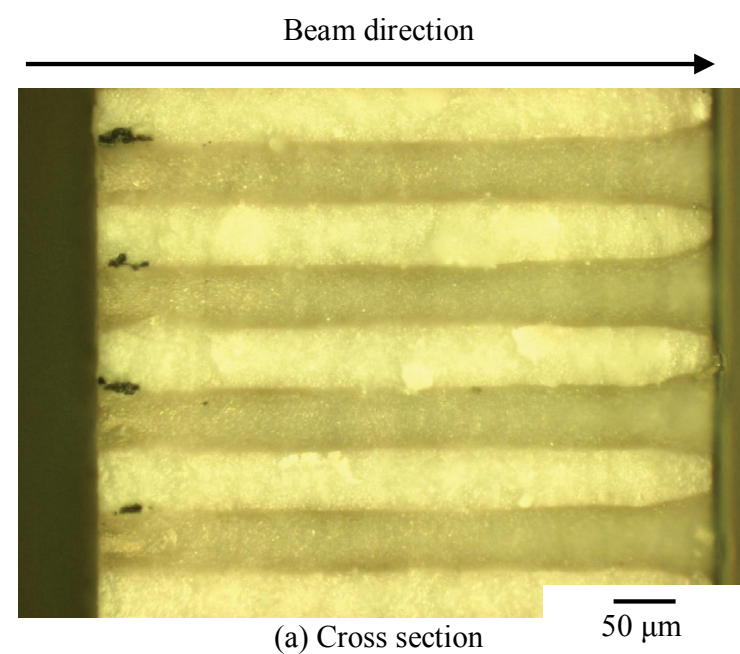

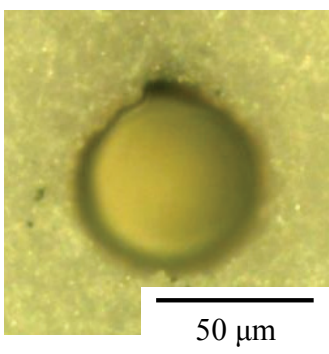

(b) Entrance hole

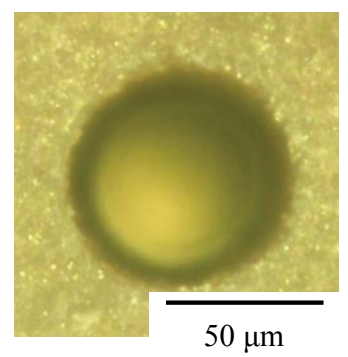

(c) Exit hole
Figure 4 Optical photographs of (a) cross section of through holes, (b) entrance hole and (c) exit hole. Through holes were formed with using PVA solution.

Beam direction

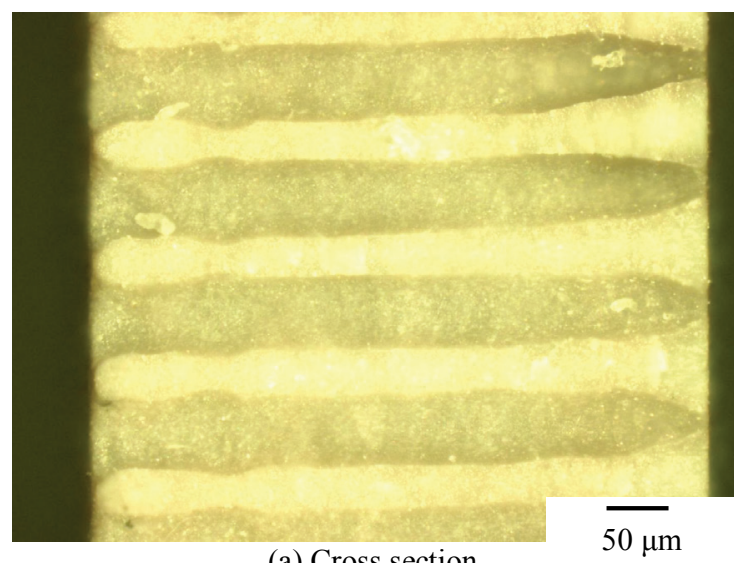

(a) Cross section

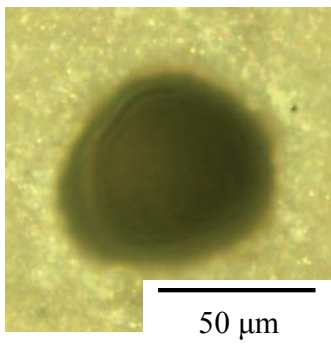

(b) Entrance hole

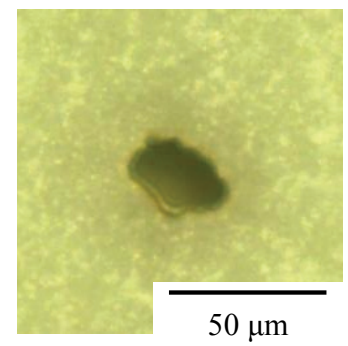

(c) Exit hole
Figure 5 Optical photographs of (a) cross section of through holes, (b) entrance hole and (c) exit hole. Through holes were formed with using tap water. energy of the laser beam was $200 \mu \mathrm{J}$, the pulse repetition rate was $5 \mathrm{kHz}$ and the number of scanning times of the laser beam along the periphery of the hole was set to 35 times. The beam scan speed was $815 \mu \mathrm{m} / \mathrm{s}$. The energy fluence of $71 \mathrm{~J} / \mathrm{cm}^{2}$ was achieved at the focal point. In the through hole drilling process, it is generally known that the diameter of the through hole on the laser beam exit side of the processing target is smaller than the incidence side, i.e., the hole is formed in a so-called tapered shape due to the characteristics of the laser beam. Figure 3 shows typical through holes having tapered shape. The entrance hole diameter is about $56 \mu \mathrm{m}$ and the exit hole diameter is about $19 \mu \mathrm{m}$. As shown in this figure, the exit hole is crooked and irregular in outline.

As mentioned previously, the substrate for the prove card need to have straight shape through holes that the probes go through with an appropriate clearance. In order to make straight through holes, a commercially available aqueous synthetic glue (hereinafter referred to as PVA glue) which is the solution of polymer material containing polyvinyl alcohol (PVA) was arranged to be in contact with the back side of machinable ceramics. The PAV glue was used as the flowable backing material after diluting 1.5 times with tap water. The cross section of through holes drilled by using PVA solution as the flowable backing material is shown in Figure 4. In both situations of Figure 3 and Figure 4, the difference among the experimental conditions is just the presence or absence of the flowable backing material. Other conditions such as the pulse energy, the pulse repetition rate, the number of scanning times of the laser beam remain the same. The focal point is kept to

Beam direction

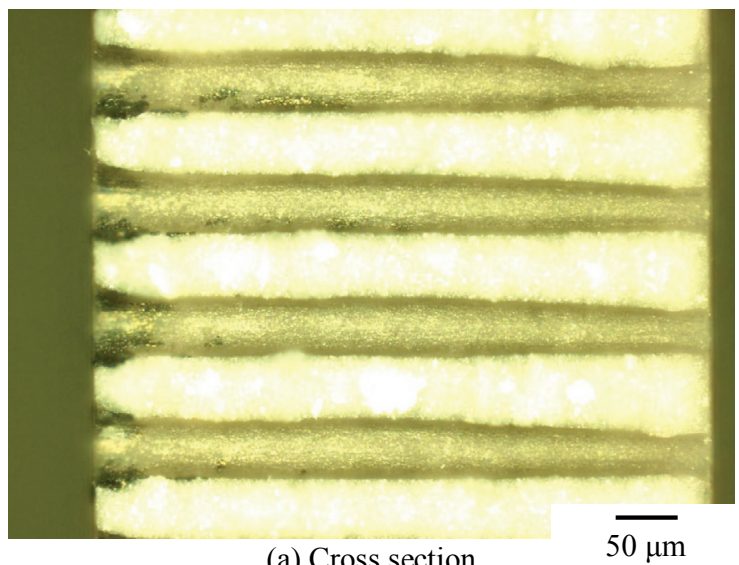

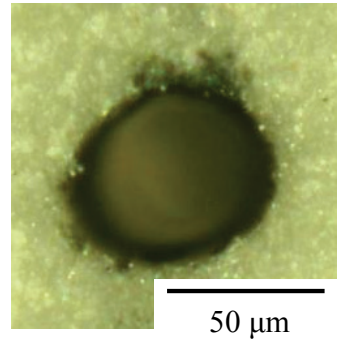

(b) Entrance hole

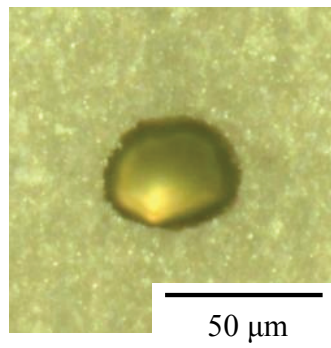

(c) Exit hole
Figure 6 Optical photographs of (a) cross section of through holes, (b) entrance hole and (c) exit hole. Through holes were formed with using glycerin. 
the front side of the target material during the UV laser radiation. It can be understood from Figure 4 that each formed through hole has a cylindrical shape although the exit hole diameter is a slightly larger than the entrance hole diameter. The entrance hole diameter is about $56 \mu \mathrm{m}$ and the exit hole diameter is about $68 \mu \mathrm{m}$. By using PAV solution as the flowable backing material, the exit hole diameter is obviously expanded and moreover, the outlines of the entrance and the exit holes are sharply defined in a circle. We consider that the exit hole diameter is able to be controlled by the number of scanning times of the laser beam along the periphery of the hole. Since the PVA glue has properties that it is readily dissolved in warm water of temperature from 40 to $80{ }^{\circ} \mathrm{C}$, PVA solution can be easily removed by cleaning the processing target with warm water after laser processing.

In order to compare the effect of PVA solution, tap water was used as the flowable backing material. Other experimental conditions are the same in the situation of Figure 4. The result is shown in Figure 5. As shown in this figure, the exit hole diameter isn't expanded although the middle portion of holes is expanded. In this case, the entrance hole diameter is about $61 \mu \mathrm{m}$ and the exit hole diameter is about $22 \mu \mathrm{m}$. Glycerin was also used as the flowable backing material in another comparative experiment. The result is shown in Figure 6. Other experimental conditions are the same with the situation of Figure 4. The entrance hole diameter is about $59 \mu \mathrm{m}$ and the exit hole diameter is about $37 \mu \mathrm{m}$. In this situation, the exit hole diameter is about two times bigger than that obtained without using the flowable backing material (as shown in Figure 3). Although the shape of the thought holes is still tapered when glycerin was used as the flowable baking material, the increase in the number of scanning times of the laser beam along the periphery of the hole has the potential of improving the through hole shape. This is because a contact time between the plasma and the flowable backing material is an important factor in this process as described later. From above results, we can speculate that there is an appropriate solution for the flowable backing material. And of course, we consider that the solution depends on the type of the target materials.

Why does the exit diameter of the through holes expand when PVA solution is arranged to be in contact with the back side of the target material during the UV laser radiation? The reason yet remained unclear, nevertheless, we consider that plasma formed as a result of the laser beam's absorption into the flowable backing material is the main cause of the phenomenon. In our previous study, the

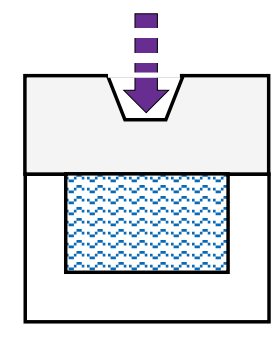

(a) Initial stage

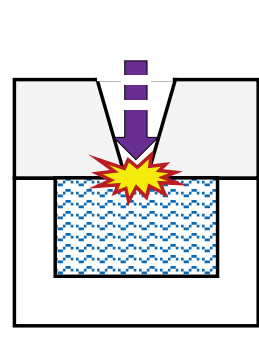

(b) Middle stage

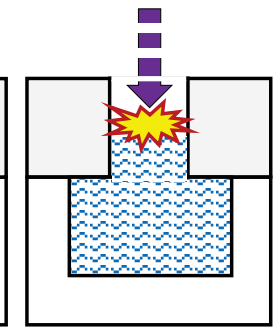

(c) Later stage
Figure 7 Elevation of plasma. laser beams of four different wavelengths, i.e., fundamental wave, second harmonic wave, third harmonic wave and fourth harmonic wave of a Q-switched Nd:YAG laser with pulse duration of $4-6 \mathrm{~ns}$ were irradiated to the resin material in order to examine the effect of the absorption rate of the laser beam to the flowable backing material [2]. PVA glue was used as the flowable backing material during the laser radiation. When fundamental wave or second harmonic wave was irradiated to the resin material, the exit diameter of the through hole did not expand even though the flowable backing material was used. In the situations of third harmonic wave and fourth harmonic wave, the exit diameter of the through hole expanded. Especially when fourth harmonic wave was irradiated, straight through hole was obtained in the resin material. From these results, we consider that the laser beams of third harmonic wave and fourth harmonic wave, i.e., UV lasers are the best for this process.

Figure 7 shows the schematic illustration of the plasma produced at the front of the laser beam. The laser beam which penetrated the processing target strikes the flowable backing material and is absorbed into that, then, the plasma including ingredient of the flowable backing material is generated there. In this study, we used UV laser having a wavelength of $266 \mathrm{~nm}$. Light absorption rate of the substances is higher in the UV region as mentioned in the previous paragraph. Therefore the superheated plasma is expected to be generated by the absorption of UV laser beam into the flowable backing material. The plasma is elevated with the flowable backing material from the backside of the target material to the front side of that by a capillary phenomenon. By the elevation of the plasma, we consider that the shape of the through holes changes from tapered shape to straight shape. Therefore the contact time between the plasma and the flowable backing material is an important factor in this process using the flowable backing material, not to mention the excited species included in the plasma.

Now we focus our attention on the existence of the hydroxyl group $(-\mathrm{OH})$ in the flowable backing material. PVA glue is the solution of polymer material containing polyvinyl alcohol. Polyvinyl alcohol has the hydroxyl group in the molecule. Glycerin also contains the hydroxyl group. We consider that the hydroxyl group reacts with UV laser beam to form hydroxyl radical [7]. In general, the hydroxyl radicals are highly reactive. Therefore, we consider that the expansion of the through hole diameter on the exit surface is caused by the hydroxyl radicals at this stage of study.

\section{Conclusions}

A new approach to the through hole forming was demonstrated to produce through holes of straight shape in machinable ceramics. The through holes having straight shape were successfully fabricated when PVA solution was arranged to be in contact with the back side of machinable ceramics during the UV laser radiation. This unique through hole forming method can easily control the shape of the through holes. By using this technique, we will be able to make the substrate for the probe card which has straight shape holes with narrow pitch. 


\section{References}

[1] S.Nakamura, K.Itagaki and N.Soma: Online Proceedings of LPM 2013, \#13-081: A132 (2013)

[2] S.Nakamura, k.Itagaki and N.Soma: JLMN-Journal of Laser Micro/Nanoengineering Vol. 9, No. 1, (2014) pp.73-78.

[3] http://www.jem-net.co.jp/en/products/pro_hando_top_ html

[4] H.Endo and E.Marui: Int J Mach Tools Manuf, Vol.46 (2006) pp.575-579.

[5] C.Mullan, D.Ilie, G.M.O'Connor, S.Favre and T.J.Glynn: Proc. SPIE, Vol. 6459, (2007) pp. 64590G.1-64590G.12.

[6]http://www.ftceramics.co.jp/eng/products/machinable/Nitride/

[7] B.R.Locke, M.Sato, P.Sunka, M.R.Hoffmann and J.S. Chang: Ind. Eng. Chem. Res., Vol.45, (2006) pp.882905.

(Received: March 31, 2014, Accepted: November 23, 2014) 